\title{
A necessary and sufficient condition for the non- emptiness of the core of a non-transferable utility game
}

Citation for published version (APA):

Predtetchinski, A., \& Herings, P. J. J. (2002). A necessary and sufficient condition for the non-emptiness of the core of a non-transferable utility game. METEOR, Maastricht University School of Business and Economics. METEOR Research Memorandum No. 019 https://doi.org/10.26481/umamet.2002019

Document status and date:

Published: 01/01/2002

DOI:

10.26481/umamet.2002019

Document Version:

Publisher's PDF, also known as Version of record

Please check the document version of this publication:

- A submitted manuscript is the version of the article upon submission and before peer-review. There can be important differences between the submitted version and the official published version of record.

People interested in the research are advised to contact the author for the final version of the publication, or visit the DOI to the publisher's website.

- The final author version and the galley proof are versions of the publication after peer review.

- The final published version features the final layout of the paper including the volume, issue and page numbers.

Link to publication

\footnotetext{
General rights rights.

- You may freely distribute the URL identifying the publication in the public portal. please follow below link for the End User Agreement:

www.umlib.nl/taverne-license

Take down policy

If you believe that this document breaches copyright please contact us at:

repository@maastrichtuniversity.nl

providing details and we will investigate your claim.
}

Copyright and moral rights for the publications made accessible in the public portal are retained by the authors and/or other copyright owners and it is a condition of accessing publications that users recognise and abide by the legal requirements associated with these

- Users may download and print one copy of any publication from the public portal for the purpose of private study or research.

- You may not further distribute the material or use it for any profit-making activity or commercial gain

If the publication is distributed under the terms of Article $25 \mathrm{fa}$ of the Dutch Copyright Act, indicated by the "Taverne" license above, 


\title{
A Necessary and Sufficient Condition for Non-emptiness of the Core of a Non-transferable Utility Game*
}

\author{
Arkadi Predtetchinski ${ }^{\dagger} \quad$ P. Jean-Jacques Herings ${ }^{\dagger}$
}

July 1,2002

\begin{abstract}
It is well-known that a transferable utility game has a non-empty core if and only if it is balanced. In the class of non-transferable utility games balancedness or the more general $\pi$-balancedness due to Billera (1970) is a sufficient, but not a necessary condition for the core to be non-empty. This paper gives a natural extension of the $\pi$-balancedness condition that is both necessary and sufficient nonemptiness of the core.
\end{abstract}

JEL classification code: $\mathrm{C} 71$.

Keywords: cooperative games, core, balancedness.

${ }^{*}$ Helpful comments of Hans Peters and Andrés Perea are gratefully acknowledged.

${ }^{\dagger}$ Department of Economics, Universiteit Maastricht, P.O. Box 616, 6200 MD Maastricht, The Netherlands. Tel.: +31-43-38883635, fax: +31-43-38884878, e-mail: a.predtetchinski@algec .unimaas.nl, p.herings@algec.unimaas.nl. 


\section{Introduction}

A cooperative game with non-transferable utility is an assignment of a set of feasible utility allocations to each coalition of players. The core of a cooperative game selects those utility allocations that are robust to all possible deviations by coalitions.

In Bondareva (1963) and in Shapley (1967) the notion of a balanced game has been introduced for games with transferable utility (TU-games). It has been demonstrated that a TU-game has a non-empty core if and only if it is a balanced game. Scarf (1967) has extended the condition of balancedness to games with non-transferable utility (NTU-games) and proved that every balanced NTU-game has a non-empty core.

Billera (1970) has further generalized the notion of a balanced game. In his work the balancedness condition is defined with respect to a system $\pi$ of coalitional vectors specifying the power or weight of each agent within every coalition. The existence of the system of the coalitional vectors $\pi$ such that the game is $\pi$-balanced suffices for the core to be non-empty. Moreover, when attention is restricted to hyperplane games, a non-empty core implies $\pi-$ balancedness for an appropriate choice of $\pi$. In general, the equivalence of $\pi$ balanced games and games with a non-empty core does not hold: an example of a game with non-empty core being not balanced for any choice of $\pi$ is given in Billera (1970). Generalizations of Billera's balancedness conditions have been given for cooperative games in permutational structure (see van der Laan, Talman and Yang (1998)) and cooperative games in graph structure (see Herings, van der Laan and Talman (2000)). These conditions weaken $\pi$-balancedness and are sufficient for the core to be non-empty, though not necessary.

A natural generalization of the $\pi$-balancedness condition is achieved by allowing the system of coalitional vectors $\pi$ to depend on the utility allocations. In this paper we define the balancedness of the game with respect to a correspondence $\Pi$ that assigns to each allocation of utilities a set of coalitional vectors $\pi$. We therefore allow the power of an agent within a coalition to depend on the utility allocation that is proposed. We prove that the core of an NTU-game is non-empty if it is balanced with respect to some correspondence $\Pi$. Conversely, for a given game with a non-empty core we construct a correspondence $\Pi$ satisfying the condition of $\Pi$-balancedness. Thus, $\Pi$-balancedness is not only a sufficient, but also a necessary condition for non-emptiness of the core. 
To the best of our knowledge, the only alternative necessary and sufficient balancedness condition for non-emptiness of the core is given by Keiding and Thorlund-Petersen (1987). The advantage of our $\Pi$-balancedness condition over the condition in Keiding and Thorlund-Peterson (1987) is that it applies directly to the game of interest and avoids the construction of any auxiliary games or sequences of approximating games.

The outline of the paper is as follows. In Section 2 some notation is introduced. In Section 3 we discuss the $\pi$-balancedness condition of Billera (1970) and the $(\pi,<)$-balancedness condition of Keiding and Thorlund-Peterson (1987). In Section 4 the $\Pi$-balancedness condition is defined and the main result of the paper is proved.

\section{Notation}

The symbol $\subseteq$ is used to denote 'inclusion', while the symbol $\subset$ is used to denote 'strict inclusion'. Let $n$ be a positive integer. Then $N$ is the set of integers $\{1, \ldots, n\}$, and $\mathcal{N}$ is the collection of non-empty subsets of the set $N$. $\mathbb{R}^{n}$ is the space of functions $x: i \in N \mapsto x^{i} \in \mathbb{R}$. $\mathbb{R}^{\mathcal{N}}$ is the space of functions $\lambda: S \in \mathcal{N} \mapsto \lambda_{S} \in \mathbb{R}$. Let $\Delta_{N}$ denote the unit simplex in $\mathbb{R}^{n}, \Delta_{N}=\left\{x \in \mathbb{R}^{n} \mid x^{i} \geq 0 \quad \forall i \in N, \sum_{i \in N} x^{i}=1\right\}$. For every $S \in \mathcal{N}$ define the set $\Delta_{S}=\left\{x \in \Delta_{N} \mid \sum_{i \in S} x^{i}=1\right\}$, and let $\Delta$ be a Cartesian product of $\Delta_{S}$ over all $S \in \mathcal{N}$. Let $\Delta_{\mathcal{N}}$ denote the unit simplex in $\mathbb{R}^{\mathcal{N}}, \Delta_{\mathcal{N}}=$ $\left\{\lambda \in \mathbb{R}^{\mathcal{N}} \mid \lambda_{S} \geq 0 \forall S \in \mathcal{N}, \sum_{S \in \mathcal{N}} \lambda_{S}=1\right\}$. If $x$ and $y$ are elements of $\mathbb{R}^{n}$, then $\langle x, y\rangle$ denotes the scalar product of $x$ and $y$, that is $\langle x, y\rangle=\sum_{i \in N} x^{i} y^{i}$. For each $S \in \mathcal{N}$ let $\mathbf{1}_{S}$ be the vector in $\mathbb{R}^{n}$ with component $\mathbf{1}_{S}^{i}$ equal to 1 if $i \in S$ and equal to 0 otherwise. For a subset $A$ of $\mathbb{R}^{n}$, the symbols int $A, \partial A$, and $\operatorname{conv} A$ denote, respectively, the interior, the boundary, and the convex hull of the set $A$.

\section{$3 \pi$-balancedness and $(\pi,<)$-balancedness}

An $n$-person game with non-transferable utility (hereafter referred to simply as a game) is a family of sets $V=\langle V(S)\rangle_{S \in \mathcal{N}}$ satisfying the following assumptions:

$(\mathcal{G} 1)$ For all $S \in \mathcal{N}, V(S)$ is a non-empty proper closed subset of $\mathbb{R}^{n}$. 
$(\mathcal{G} 2)$ For all $S \in \mathcal{N},\left[x \in V(S), \quad y \in \mathbb{R}^{n}, \quad y^{i} \leq x^{i}\right.$ for all $\left.i \in S\right]$ implies $[y \in V(S)]$.

(G3) The set $V(N) \backslash \bigcup_{i \in N} i n t V(\{i\})$ is non-empty and compact.

A vector $x \in \mathbb{R}^{n}$ is an element of the core of the game $V$ if $x \in V(N)$ and there exist no $S \in \mathcal{N}$ and no $y \in V(S)$ such that $x^{i}<y^{i}$ for all $i \in S$. The core of the game $V$ is denoted by $C(V)$. Therefore,

$$
C(V)=V(N) \backslash \bigcup_{S \in \mathcal{N}} \operatorname{int} V(S) .
$$

Below we reproduce the $\pi$-balancedness condition of Billera (1970). Let an element $\pi$ of the set $\Delta$ be given. A collection of coalitions $\beta \subseteq \mathcal{N}$ is said to be $\pi$-balanced if there exists $\lambda \in \Delta_{\mathcal{N}}$ such that

$$
\pi_{N}=\sum_{S \in \beta} \lambda_{S} \pi_{S}
$$

A game $V$ is said to be $\pi$-balanced if for every $\pi$-balanced collection of coalitions $\beta \subseteq \mathcal{N}$ it holds that

$$
\bigcap_{S \in \beta} V(S) \subseteq V(N)
$$

If there exists some $\pi \in \Delta$ such that the game $V$ is $\pi$-balanced, then the core of $V$ is non-empty.

The $\pi$-balancedness condition has been generalized in the work of Keiding and Thorlund-Peterson (1987). Given a game $V$ and a coalition $S \in \mathcal{N}$ let $V_{p}(S)=\left\{x^{S} \in \mathbb{R}_{+}^{S} \mid x \in V(S)\right\}$, where $\mathbb{R}_{+}^{S}$ is the set of non-negative functions with the domain $S$, and $x^{S}$ is the restriction of the function $x \in \mathbb{R}^{n}$ to the set $S .{ }^{1}$ Let $\mathcal{R}$ denote the set of binary relations $>$ on the set $\mathcal{N}$ satisfying the following conditions:

- > is acyclic: If $S_{j} \in \mathcal{N}$ for all $j \in\{1, \ldots, k\}$ and $S_{1}<S_{2}<\cdots<S_{k}$, then we cannot have $S_{k}<S_{1}$;

\footnotetext{
${ }^{1}$ In the paper of Keiding and Thorlund-Peterson (1987) a somewhat different definition of a non-transferable utility game is given. In particular, the set $V(S)$ is assumed to lie entirely in the non-negative orthant of $\mathbb{R}^{n}$ and to contain zero vector. The set $V_{p}(S)$ is assumed to be bounded for all $S \in \mathcal{N}$.
} 
- all one player coalitions are minimal: For all $i \in N$ there is no $S \in \mathcal{N}$ such that $S<\{i\}$.

Let $\pi \in \Delta$ and $<\in \mathcal{R}$ be given. A game $V$ is said to be $(\pi,<)$-balanced if the following condition is satisfied: If $\beta \subseteq \mathcal{N}$ is a $\pi$-balanced collection of coalitions and

$$
x \in \bigcap_{S \in \beta} V(S) \backslash \bigcup_{Q \in \mathcal{N},} \bigcup_{T \in \beta: Q<T} \operatorname{int} V(Q),
$$

then $x \in V(N)$. The game $V$ is said to be weakly $(\pi,<)$-balanced if there exists a sequence $\left\{V^{\tau}\right\}_{\tau=1}^{\infty}$ of $(\pi,<)$-balanced games such that

- $V(N)=V^{\tau}(N)$ for all $\tau$, and

- for every $S \in \mathcal{N}$ the sequence $\left\{V_{p}^{\tau}(S)\right\}_{\tau=1}^{\infty}$ converges to the set $V_{p}(S)$ in the topology induced by the Hausdorff metric on the set of non-empty compact subsets of $\mathbb{R}^{n}$.

Theorem 1 below gives a characterization of NTU-games with a non-empty core.

Theorem 1 (Keiding and Thorlund-Peterson, 1987) Let $V$ be a game. Then the core of $V$ is non-empty if and only if there exist $\pi \in \Delta,<$ $\in \mathcal{R}$, and a weakly $(\pi,<)$-balanced game $V^{\prime}$ such that $V(N)=V^{\prime}(N)$ and $V(S) \subseteq V^{\prime}(S)$ for all $S \in \mathcal{N}$.

The purpose of the next section is to give a necessary and sufficient condition for non-emptiness of the core that applies directly to the game of interest $V$. In particular, our condition of $\Pi$-balancedness avoids the construction of an auxiliary game like $V^{\prime}$ and does not use the approximation of the game $V$ by a sequence of games $\left\{V^{\tau}\right\}_{\tau=1}^{\infty}$.

\section{4 П-balancedness}

As a preliminary result we state the $\Pi$-balanced version of the weak Knaster, Kuratowski, Mazurkiewicz, and Shapley $(\mathrm{K}-\mathrm{K}-\mathrm{M}-\mathrm{S})$ theorem. The original version of the K-K-M-S theorem can be found in Shapley (1973). Theorem 1 can be easily established using the fixed point theorem of Kakutani (analogous to the proof of the K-K-M-S theorem in Herings (1997)). 
Theorem 2 (П-balanced weak $\mathbf{K}-\mathbf{K}-\mathbf{M}-\mathbf{S})$ Let $\left\{C_{S} \subseteq \Delta_{N} \mid S \in \mathcal{N}\right\}$ be a closed cover of $\Delta_{N}$ such that if $S$ and $T$ are elements of $\mathcal{N}$ and the set $\Delta_{T} \cap C_{S}$ is non-empty, then $S \subseteq T$. Let $\Pi: \Delta_{N} \mapsto \Delta$ be a convexvalued upper hemicontinuous correspondence. Then there exist $x^{*} \in \Delta_{N}$, $\pi^{*} \in \Pi\left(x^{*}\right)$, and $\lambda^{*} \in \Delta_{\mathcal{N}}$ such that

$$
\begin{aligned}
& x^{*} \in \bigcap_{S \in \mathcal{N}: \lambda_{S}^{*}>0} C_{S} \\
& \pi_{N}^{*}=\sum_{S \in \mathcal{N}} \lambda_{S}^{*} \pi_{S}^{*} .
\end{aligned}
$$

Definition 1 Let a game $V$ and a convex-valued upper hemicontinuous correspondence $\Pi: \mathbb{R}^{n} \mapsto \Delta$ be given. The game $V$ is said to be $\Pi$-balanced if the following condition is satisfied: If $x \in \mathbb{R}^{n}, \pi \in \Pi(x)$, and $\lambda \in \Delta_{\mathcal{N}}$ are such that

$$
\begin{gathered}
x \in \bigcap_{S \in \mathcal{N}: \lambda_{S}>0} V(S), \\
\pi_{N}=\sum_{S \in \mathcal{N}} \lambda_{S} \pi_{S},
\end{gathered}
$$

then $x \in V(N)$.

The $\pi$-balancedness condition of Billera (1970) is defined relative to a fixed element $\pi$ of the set $\Delta$. It is a special case of Definition 1 that allows $\pi$ to depend upon the utility allocations via the correspondence $\Pi$. By using the concept of $\Pi$-balancedness, we allow the power of an agent within a coalition to depend on the utility allocation that is proposed.

Theorem 3 Let $V$ be a game. Suppose that there exists a convex-valued upper hemicontinuous correspondence $\Pi: \mathbb{R}^{n} \mapsto \Delta$ such that the game $V$ is $\Pi$-balanced. Then the core of the game $V$ is non-empty.

We do not report the proof of Theorem 3. Our argument essentially replicates the proof of the Scarf's theorem given in Shapley (1973) and in Kannai (1992). The key role in the proof of Theorem 3 is played by the $\Pi$-balanced weak $\mathrm{K}-\mathrm{K}-\mathrm{M}-\mathrm{S}$ theorem.

Theorem 4 below asserts that $\Pi$-balancedness is not only a sufficient, but also a necessary condition for the non-emptiness of the core. 
Theorem 4 Let $V$ be a game with a non-empty core. Then there exists a convex-valued upper hemicontinuous correspondence $\Pi: \mathbb{R}^{n} \mapsto \Delta$ such that the game $V$ is $\Pi$-balanced.

Proof. We make use of the following lemma.

Lemma 1 Let $V$ be a game and let $S \in \mathcal{N}$. Then

1. for every $x \in \mathbb{R}^{n}$ there exists a unique real number $t_{S}(x)$ such that $\left(x-t_{S}(x) \mathbf{1}_{S}\right) \in \partial V(S)$;

2. the function $t_{S}: x \mapsto t_{S}(x)$ is a continuous function;

3. $x \in V(S)$ if and only if $t_{S}(x) \leq 0 ; x \in \mathbb{R}^{n} \backslash \operatorname{int} V(S)$ if and only if $t_{S}(x) \geq 0$.

Define the continuous mapping $g_{S}: \mathbb{R}^{n} \rightarrow \partial V(S)$ by $g_{S}(x)=x-t_{S}(x) \mathbf{1}_{S}$ for all $x \in \mathbb{R}^{n}$.

Let $x^{*}$ be an element of the core of the game $V$. Define the correspondence $\Pi: \mathbb{R}^{n} \rightarrow \Delta$ to be the Cartesian product over all $S \in \mathcal{N}$ of the correspondences $\Pi_{S}: \mathbb{R}^{n} \rightarrow \Delta_{S}$, where

$$
\Pi_{S}(x)=\left\{\pi_{S} \in \Delta_{S} \mid\left\langle\pi_{S}, g_{S}\left(x^{*}\right)\right\rangle=\left\langle\pi_{S}, g_{S}(x)\right\rangle\right\}
$$

for all $x \in \mathbb{R}^{n}$.

To see that the set $\Pi_{S}(x)$ is non-empty, observe that it is given by the intersection of the sets $\Pi_{S}^{-}(x)$ and $\Pi_{S}^{+}(x)$, where

$$
\begin{aligned}
& \Pi_{S}^{-}(x)=\left\{\pi_{S} \in \Delta_{S} \mid\left\langle\pi_{S}, g_{S}\left(x^{*}\right)\right\rangle \leq\left\langle\pi_{S}, g_{S}(x)\right\rangle\right\}, \\
& \Pi_{S}^{+}(x)=\left\{\pi_{S} \in \Delta_{S} \mid\left\langle\pi_{S}, g_{S}\left(x^{*}\right)\right\rangle \geq\left\langle\pi_{S}, g_{S}(x)\right\rangle\right\} .
\end{aligned}
$$

If the set $\Pi_{S}^{-}(x)$ were empty for some $x \in \mathbb{R}^{n}$, then the inequality $\left\langle\pi_{S}, g_{S}\left(x^{*}\right)\right\rangle>$ $\left\langle\pi_{S}, g_{S}(x)\right\rangle$ would be satisfied for all $\pi_{S} \in \Delta_{S}$. In particular, this inequality would hold for all vertices of the simplex $\Delta_{S}$, implying that $g_{S}^{i}\left(x^{*}\right)>g_{S}^{i}(x)$ for all $i \in S$. As $g_{S}\left(x^{*}\right) \in \partial V(S)$, this would imply $g_{S}(x) \in \operatorname{int} V(S)$, contradicting the definition of $g_{S}$. A similar argument shows that the set $\Pi_{S}^{+}(x)$ is non-empty. Moreover, both sets $\Pi_{S}^{-}(x)$ and $\Pi_{S}^{+}(x)$ are closed, and $\Pi_{S}^{-}(x) \cup \Pi_{S}^{+}(x)=\Delta_{S}$. Connectedness of the set $\Delta_{S}$ implies that the intersection of $\Pi_{S}^{-}(x)$ and $\Pi_{S}^{+}(x)$ is non-empty. 
Convexity of the sets $\Pi_{S}(x)$ is trivial.

To prove the upper hemicontinuity of $\Pi$, let $\left(x_{(k)}, \pi_{(k)}\right)$ be a sequence of points in $\mathbb{R}^{n} \times \Delta$ satisfying $\pi_{(k)} \in \Pi\left(x_{(k)}\right)$ and converging to some point $\left(x_{(0)}, \pi_{(0)}\right)$. Then the equalities

$$
\left\langle\pi_{S(k)}, g_{S}\left(x^{*}\right)\right\rangle=\left\langle\pi_{S(k)}, g_{S}\left(x_{(k)}\right)\right\rangle, S \in \mathcal{N}
$$

hold for all members of the sequence. By continuity of the mappings $g_{S}$, it holds in the limit that

$$
\left\langle\pi_{S(0)}, g_{S}\left(x^{*}\right)\right\rangle=\left\langle\pi_{S(0)}, g_{S}\left(x_{(0)}\right)\right\rangle, S \in \mathcal{N} .
$$

Therefore, $\pi_{(0)} \in \Pi\left(x_{(0)}\right)$.

Next we show that the game $V$ is $\Pi$-balanced. Let $x \in \mathbb{R}^{n}, \pi \in \Pi(x)$, and $\lambda \in \Delta_{\mathcal{N}}$ be such that

$$
\begin{array}{r}
x \in \bigcap_{S \in \mathcal{N}:} V(S) \\
\pi_{N}=\sum_{S \in \mathcal{N}} \lambda_{S} \pi_{S} .
\end{array}
$$

By part (3) of Lemma 1, for all $S \in \mathcal{N}$ with $\lambda_{S}>0$ it holds that $t_{S}(x) \leq 0$. As $x^{*} \notin \operatorname{int} V(S)$, we have $0 \leq t_{S}\left(x^{*}\right)$. It follows that for all $S \in \mathcal{N}$ with $\lambda_{S}>0$ the following inequalities hold:

$$
\begin{aligned}
\left\langle\pi_{S}, x\right\rangle & \leq\left\langle\pi_{S}, g_{S}(x)\right\rangle \\
& =\left\langle\pi_{S}, g_{S}\left(x^{*}\right)\right\rangle \\
& \leq\left\langle\pi_{S}, x^{*}\right\rangle .
\end{aligned}
$$

Therefore,

$$
\begin{aligned}
\left\langle\pi_{N}, x\right\rangle & =\sum_{S \in \mathcal{N}: \lambda_{S}>0} \lambda_{S}\left\langle\pi_{S}, x\right\rangle \\
& \leq \sum_{S \in \mathcal{N}: \lambda_{S}>0} \lambda_{S}\left\langle\pi_{S}, x^{*}\right\rangle \\
& =\left\langle\pi_{N}, x^{*}\right\rangle \\
& =\left\langle\pi_{N}, g_{N}(x)\right\rangle,
\end{aligned}
$$

where the last equality is implied by the fact that $x^{*}=g_{N}\left(x^{*}\right)$ and by definition of $\Pi_{N}$. By definition of $g_{N}(x)$, this gives the inequality $t_{N}(x) \leq 0$, and the inclusion $x \in V(N)$. 
Theorems 3 and 4 show that $\Pi$-balancedness is necessary and sufficient for non-emptiness of the core. The condition is relatively simple and provides a natural extension of $\pi$-balanced concept of Billera that closes the gap between necessary and sufficient conditions for non-emptiness of the core.

\section{References}

[1] Billera, L.J. (1970): "Some Theorems on the Core of an $n$-Person Game Without Side-Payments," SIAM Journal of Applied Mathematics, 18, $567-579$.

[2] Bondareva, O.N. (1963): "Some Applications of Linear Programming Methods to the Theory of Cooperative Games," Problemy Kibernetiki, 10, 119-139 [in Russian].

[3] Herings, P.J.J. (1997): "An Extremely Simple Proof of the K-K-M-S Theorem," Economic Theory, 10, 361-367.

[4] Herings, P.J.J., G. van der Laan, and A.J.J. Talman (2000), "Cooperative Games in Graph Structure," METEOR Research Memorandum 00/26, University of Maastricht, pp. 1-30.

[5] Kannai, Y. (1992): "The Core and Balancedness," in Handbook of Game Theory with Economic Applications, Chapter 12 (eds. R.J. Aumann and S. Hart). Amsterdam: North-Holland.

[6] Keiding, H. and L. Thorlund-Petersen (1987): "The Core of a Cooperative Game without Side Payments," Journal of Optimization Theory and Applications, 54, 273-288.

[7] Laan, van der G., A.J.J. Talman, and Z. Yang (1998): "Cooperative Games in Permutational Structure," Economic Theory 11, 427-442.

[8] Scarf, H. (1967): "The Core of an n-Person Game," Econometrica 35, 50-69.

[9] Shapley, L.S. (1967): "On Balanced Sets and Cores," Navel Research Logistics Quartely 14, 453-460. 
[10] Shapley, L.S. (1973): "On Balanced Games without Side Payments," in Mathematical programming, pp. 261-290 (eds. T.C. Hu and S.M. Robinson). New-York: Academic Press. 